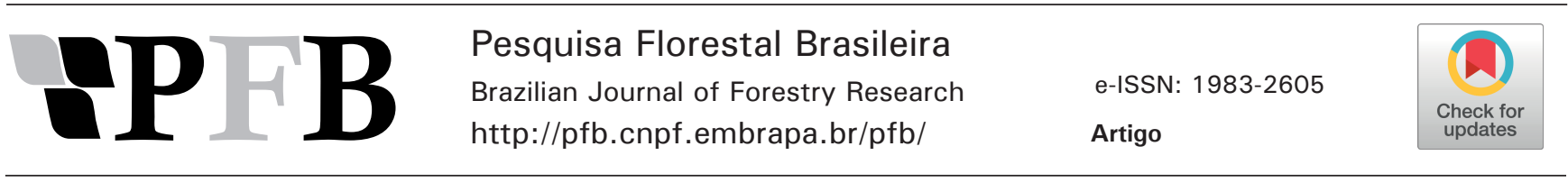

\title{
Detecção automática de árvores em unidades amostrais a partir de varredura simples com laser terrestre
}

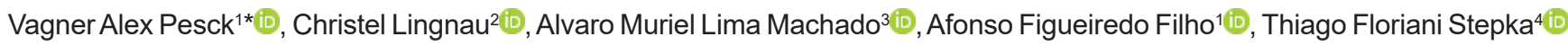 \\ ${ }_{1}^{1}$ Universidade Estadual do Centro Oeste, Departamento de Engenharia Florestal, km 07, BR 153, Riozinho, CEP 84500-000, Irati, PR, Brasil \\ ${ }^{2}$ Universidade Federal do Paraná, Departamento de Ciências Florestais, Av. Prefeito Lothário Meissner, 900, Jardim Botânico, CEP 80210-170, Curitiba, PR, Brasil \\ 3Universidade Federal do Paraná, Departamento de Geomática, R. Cel. Francisco Herácito dos Santos, s/n, CEP 81531-990, Jardim das Américas, Curitiba, PR, Brasil \\ ${ }^{4}$ Universidade do Estado de Santa Catarina, Departamento de Engenharia Florestal, Av. Luiz de Camões, 2090, Conta Dinheiro, CEP 88520-000, Lages, SC, Brasil
}

"Autor correspondente:

vapesck@unicentro.br

Termos para indexação:

Sombreamento

Eucalyptus

Relevo

Index terms:

Shading

Eucalyptus

Relief

Histórico do artigo:

Recebido em 03/09/2018

Aprovado em 21/05/2019

Publicado em 31/12/2019
Resumo - O objetivo deste estudo foi a identificação automática de árvores em unidades amostrais circulares de 200, 300 e $400 \mathrm{~m}^{2}$ em povoamentos de Eucalyptus spp. a partir de dados de TLS (Terrestrial Laser Scanning) no estado do Mato Grosso do Sul. Foram testadas três idades de plantio: árvores com 2 anos, sendo consideradas com desrama natural (E. urophylla) e sem desrama natural (E. grandis); árvores com 4,5 e com 5,5 anos, sem desrama (hibrido E. urophylla x E. grandis). A varredura laser em campo foi realizada com o equipamento Trimble TX5. Para a identificação automática das árvores, foi realizado um recorte na nuvem de pontos a 1,30 m do solo (DAP) e desenvolvido o algoritmo denominado fatia 2D. Foi possível a detecção de 98,3, 98 e 93,9\% das árvores para as unidades amostrais de 200, 300 e $400 \mathrm{~m}^{2}$, respectivamente. A distribuição espacial das árvores no plantio influenciou a identificação, ocasionando sombreamento das árvores mais distantes do equipamento laser. A adoção de varreduras simples deve ser aplicada somente em unidades amostrais pequenas, pois unidades amostrais acima de $200 \mathrm{~m}^{2}$ acarretam maiores erros de detecção de árvores, o que pode gerar informações equivocadas nas estimativas dendrométricas da floresta.

\section{Automatic tree detection in sample plots from a simple terrestrial laser scanning}

\begin{abstract}
This study aimed the automatic identification of trees in circular sample units of 200, 300 and $400 \mathrm{~m}^{2}$ in stands of Eucalyptus spp., using TLS (Terrestrial Laser Scanning) in Mato Grosso do Sul State, Brazil. Three planting ages were tested: 2 years-old-plantation, considering trees with natural pruning (E. urophylla $-200 \mathrm{~m}^{2}$ ) and without natural pruning (E. grandis $\left.-300 \mathrm{~m}^{2}\right)$, and trees with 4.5 and with 5.5 years-old without pruning (hibrid E. urophylla $x$ E. grandis $-400 \mathrm{~m}^{2}$ ). Field laser scanning was performed with Trimble TX5 equipment. This approach was based on a cut of pointcloud at $1.3 \mathrm{~m}$ above ground level (DBH) and the developing the algorithm 2D slice. It was possible to detect $98.3,98$ and $93.9 \%$ of trees for samples units of 200,300 and $400 \mathrm{~m}^{2}$, respectively. These identifications were influenced by the spatial distribution of trees because of the shading of trees further from the equipment. This simple scan should be applied only for small sample units. The error detections in areas greater than $200 \mathrm{~m}^{2}$, may influence negatively the dendrometric estimations.
\end{abstract}

Trabalho apresentado no V Seminário de Atualização Florestal, 24 e 28 de setembro de 2018, Irati, PR. 


\section{Introdução}

A varredura laser terrestre (TLS) oferece uma série de vantagens sobre as medições tradicionais utilizadas em inventário florestal. Além da rápida aquisição e representação dos dados (Thies \& Spiecker, 2004), o TLS permite a extração de importantes variáveis dendrométricas como área transversal (Király \& Brolly, 2010), diâmetros a diferentes alturas (Lingnau et al., 2009, 2010; Silva, 2011; Buck, 2012; Martins Neto et al., 2013) e volume total (Buck et al., 2012) sem a necessidade do corte das árvores (Bienert et al., 2006).

O TLS tem aplicação como fonte de dados para o inventário florestal (Simonse et al., 2003; Henning \& Radtke, 2006; Liang et al., 2013; Trochta et al., 2013; Zasada et al., 2013), possibilitando uma alternativa eficiente aos métodos tradicionais de mensuração florestal.

Thies \& Spiecker (2004), utilizando um algoritmo denominado "Fatia 2D", identificaram automaticamente de 22 a 52\% das árvores com uso de varredura simples e múltiplas, respectivamente, para unidades de $900 \mathrm{~m}^{2} \mathrm{em}$ floresta composta por Faia (Fagus sylvatica L.) (95\%), e uma porção de 5\% de carvalho (Quercus spp.).

Com base em um algoritmo para a detecção de árvores, oriundas de varreduras simples, Liang et al. (2012) mapearam corretamente $73 \%$ das árvores presentes em unidades amostrais circulares com $10 \mathrm{~m}$ de raio $\left(314,16 \mathrm{~m}^{2}\right)$ em florestas boreais da Finlândia, com densidade de 1022 árvores.ha ${ }^{-1}$. Cabe destacar que a estrutura de florestas boreais é diferente de plantios florestais no Brasil, onde as árvores são plantadas em linhas e com espaçamentos regulares. Em florestas na Finlândia, Liang \& Hyypä (2013) detectaram 96\% das árvores na parcela. Os mesmos autores corroboram que varreduras múltiplas mostram ser mais precisas que varreduras simples, porém com maior tempo de processamento, devido ao tempo de deslocamento para os diferentes posicionamentos, e também com relação ao registro das nuvens de pontos a qual a varredura múltipla exige.

Considerando a importância da detecção automática de árvores para fins de inventário florestal, este estudo teve como objetivo implementar um algoritmo aplicado em unidades amostrais em plantios de Eucalyptus spp., utilizando dados de varredura simples. Adicionalmente, verificou-se a hipótese de que o método convencional e a varredura laser não diferem estatisticamente.

\section{Material e métodos}

A pesquisa foi realizada em áreas de plantios clonais de Eucalyptus spp. no município de Três Lagoas, MS de propriedade da empresa Eldorado S.A, com espaçamento de 3,6 $\mathrm{m}$ x 2,2 $\mathrm{m}$, localizadas em relevo plano a suave ondulado. No relevo plano as linhas de plantio estão perpendiculares às estradas e no relevo suave ondulado as linhas foram instaladas em curvas de nível.

A coleta de dados contemplou plantios com idades de 2 anos (com e sem desrama), e 4,5 e 5,5 anos sem desrama. O povoamento de Eucalyptus grandis Hill ex. Maiden com dois anos sem desrama foi considerado na análise dos dados para avaliar se há interferência dos galhos na identificação das árvores.

Foram instaladas e mensuradas quatro unidades amostrais por idade (Tabela 1).

Tabela 1. Dados das unidades amostrais de Eucalyptus spp. com diferentes idades no município de Três Lagoas, MS.

Table 1. Sample units data for Eucalyptus spp. with different ages in the municipality of Três Lagoas, MS.

\begin{tabular}{ccc}
\hline Espécie & Idade (anos) & $\begin{array}{c}\text { Identificação da } \\
\text { unidade amostral }\end{array}$ \\
\hline $\begin{array}{c}\text { Eucalyptus grandis } \\
\text { Eucalyptus urophylla }\end{array}$ & 2,0 & 1 a 4 \\
$\begin{array}{c}\text { Hibrido E. urophylla } x \text { E. } \\
\text { grandis }\end{array}$ & 2,0 & 5 a 8 \\
$\begin{array}{c}\text { Hibrido E. urophyllax } E . \\
\text { grandis }\end{array}$ & 5,5 & 9 a 12 \\
\hline
\end{tabular}

A distribuição das unidades amostrais circulares na área foi efetuada de forma aleatória em cada talhão, sendo as árvores da borda demarcadas para estabelecimento do limite da unidade amostral.

A varredura laser foi realizada com o equipamento Trimble TX5, baseado na diferença de fase. Tal princípio considera que a diferença entre os sinais transmitidos e refletidos, da parte fracional do comprimento total, é menor que o valor da parte inteira do comprimento de onda modulada (Maia, 1999). O campo de visão do equipamento é de $310^{\circ}$ na vertical e $360^{\circ}$ na horizontal. A resolução da varredura foi fixada em $1 / 4$ (244.000 pontos por segundo), correspondendo a uma resolução angular de $0,036^{\circ}$ e uma resolução espacial de $6,283 \mathrm{~mm}$ para uma distância de $10 \mathrm{~m}$ do dispositivo (Tabela 2). 
Tabela 2. Especificações técnicas do Trimble TX5.

Table 2. Trimble TX5 technical specifications.

\begin{tabular}{lc}
\hline \multicolumn{1}{c}{ Especificações } & Trimble TX 5 \\
Alcance de medição $(\mathrm{m})$ & De 0,6 até 120 \\
Acurácia de medição de distância até $25 \mathrm{~m}(\mathrm{~mm})$ & + ou -2 \\
Diâmetro do feixe de saída $(\mathrm{mm})$ & 3 \\
Máxima resolução $\left(^{\circ}\right)$ & 0,009 x 0,009 \\
\hline
\end{tabular}

Para a realização da varredura laser, foram estabelecidos três tamanhos de unidades amostrais, concêntricas, circulares: 200, 300 e $400 \mathrm{~m}^{2}$ (Figura 1). O equipamento laser foi posicionado no centro da unidade amostral $\left(\mathrm{X}_{0}, \mathrm{Y}_{0}, \mathrm{Z}_{0}\right)$, e o recorte dos três tamanhos foi realizado a partir deste ponto.

Para a detecção automática dos indivíduos foi observada a quantidade de árvores em pé que pode ser reconhecida a partir de uma varredura. Para a varredura a partir do ponto central da unidade amostral, foi realizado um recorte em torno da posição da leitura central. Neste caso, as árvores identificadas pela interpretação a partir de dados TLS foram quantificadas e os resultados foram comparados com o número de árvores observado nas unidades amostrais pelo inventário convencional. As etapas de processamento para identificação das árvores podem ser vistas na Figura 2.
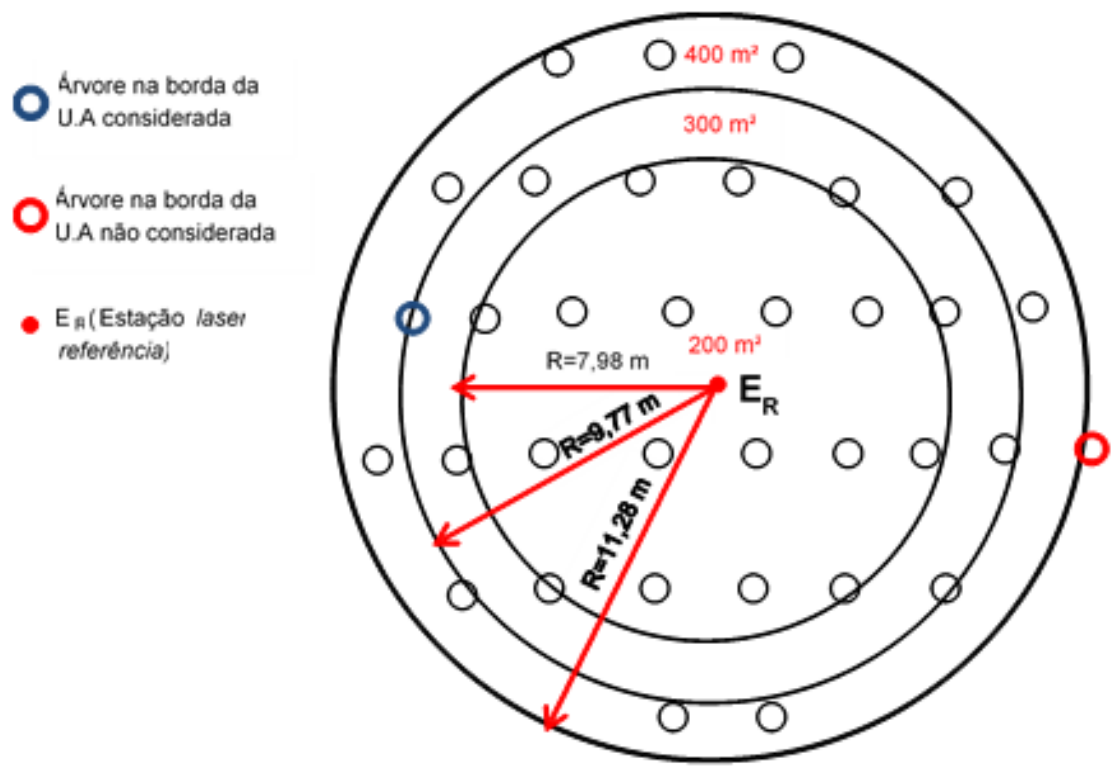

Figura 1. Delimitação das três unidades amostrais testadas para a detecção automática de árvores de Eucalyptus spp. em plantios localizados em Três Lagoas, MS.

Figure 1. Delimitation of the sample units tested for automatic detection of Eucalyptus spp. in stands located in Três Lagoas, MS.

Figura 2. Fluxograma da etapa de processamento dos dados para deteç̧ão automática de árvores em plantios de Eucalyptus spp.

Figure 2. Data processing step flowchart for automatic tree detection in plantations of Eucalyptus spp.

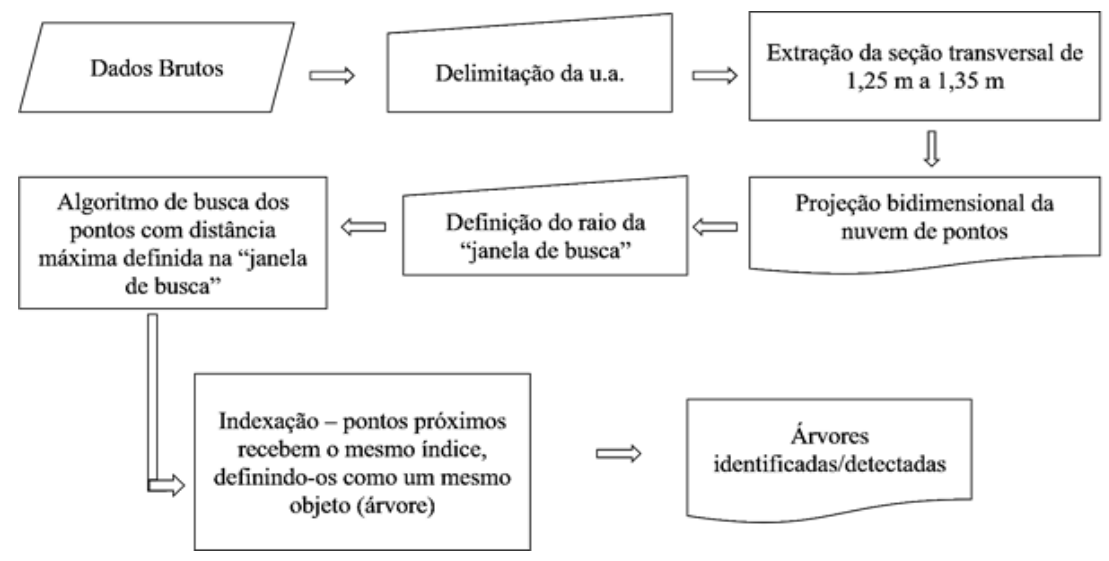

Pesq. flor. bras., Colombo, v. 39, e2018001730, p. 1-11, 2019 
O algoritmo de detecção desenvolvido na linguagem JAVA pode ser definido como:

- Delimitação da unidade amostral U.A): Recorte da nuvem de pontos ( $\mathrm{R}=7,98,9,77,11,28 \mathrm{~m}) \mathrm{e}$ da seção transversal (DAP) entre 1,25-1,35 m.

- Seleção do ponto inicial para o algoritmo de detecção automática $=\mathrm{X}_{0}, \mathrm{Y}_{0}$.

- Região de busca de agrupamento de pontos: $\left[\mathrm{X}_{0}\right.$ $\left.-\mathrm{X}_{\text {min }}, \mathrm{Y}_{0-} \mathrm{Y}_{\min }\right]$ a $\left[\mathrm{X}_{0}+\mathrm{X}_{\text {min }}, \mathrm{Y}_{0+} \mathrm{Y}_{\text {min }}\right]$, em que $\mathrm{X}_{\min }=\mathrm{Y}_{\min }=1,5 \mathrm{~m}$.

A detecção de árvores se baseia no pressuposto que as maiores densidades de pontos estão localizadas nos troncos das árvores, sendo esta a condição para considerar o objeto como árvore. Assim, o algoritmo de detecção de árvores forma um reticulado em toda a área da unidade amostral. Cada célula deste reticulado possui 1,50 por $1,50 \mathrm{~m}$, onde foi realizada a busca de pontos, sendo que esta distância é definida em função do espaçamento do plantio.

Uma seção com espessura de $0,10 \mathrm{~m}$ foi extraída da nuvem de pontos na altura entre 1,25 a $1,35 \mathrm{~m}$. $\mathrm{O}$ conjunto de pontos que representam uma árvore apareceram como semicírculo dentro de cada célula do reticulado, pois a varredura foi realizada apenas com uma visada. Os pontos da seção transversal $(0,10 \mathrm{~m})$ foram projetados para um plano cartesiano X, Y (Maas et al., 2008). A presença de um agrupamento de pontos formando um semicírculo até a distância máxima de 1,50 m foi adotada como critério para a localização de uma árvore.

Após a detecção automática, as coordenadas X e Y de cada árvore foram indexadas e salvas em um arquivo de saída e, assim, o número de árvores foi quantificado. Com as árvores detectadas nos três tamanhos de unidades amostrais, procedeu-se à validação do procedimento, a fim de verificar a existência de tendenciosidade.

Para a comparação entre o número de árvores obtido em campo e das árvores detectadas pelo algoritmo, utilizou-se o teste de identidade L\&O, proposto por Leite \& Oliveira (2002), cujo procedimento resulta da combinação do teste $\mathrm{F}\left(\mathrm{FH}_{0}\right)$ de Graybill (Graybill, 1976)
(Equação 1), do teste $t$ de Student para erro-médio ( $\left.\mathrm{t}_{\overline{\mathrm{e}}}\right)$ e da análise de correlação linear $\left(\mathrm{r}_{\mathrm{yjy} 1}\right)$ entre os valores observados $\left(\mathrm{Y}_{\mathrm{j}}\right)$ e os valores estimados $\left(\mathrm{Y}_{1}\right)$. O teste compara o valor observado como variável dependente e o valor estimado como variável independente.

$$
F\left(H_{0}\right)=\frac{(\beta-\theta)\left(Y_{1} Y_{1}\right)(\beta-\theta)}{2 R M S} \sim F_{\alpha(2, n-2 g . l .)}
$$

Onde:

$$
\begin{aligned}
& \hat{\beta}=\left[\begin{array}{l}
\hat{\beta}_{0} \\
\hat{\beta}_{1}
\end{array}\right] \quad \begin{array}{l}
=\text { Vetor de coeficientes da equação } \\
\text { de regressão. }
\end{array} \\
& \theta=\left[\begin{array}{l}
0 \\
1
\end{array}\right] ;\left(X^{\prime} X\right)^{-1}=\left[\begin{array}{cc}
n & \sum x \\
\sum x & \sum^{2} x^{2}
\end{array}\right]^{-1} \\
& \mathrm{~F}_{t a b}=[5 \% ; 2, \mathrm{n}-2 \mathrm{gl}] .
\end{aligned}
$$

As regras de decisão para a comparação entre o número de árvores observado e o identificado pelo algoritmo são apresentadas na Tabela 3. O nível de significância adotado, em todos os casos, foi $5 \%$.

Quando o erro médio não é significativo, o valor da correlação do coeficiente deve ser mais alto que $(1-|\mathrm{e}|)$ e $\mathrm{F}\left(\mathrm{H}_{0}\right)$ deve ser não significativo para que as médias sejam consideradas estatisticamente iguais (Leite \& Oliveira, 2002). Os testes F de Graybill e $t$ de Student também foram avaliados separadamente, para verificar se existem diferenças significativas entre os tratamentos.

Tabela 3. Regra de decisão para comparação entre os diferentes tamanhos de unidades amostrais.

Table 3. Decision rules for comparison between different sample unit sizes.

\begin{tabular}{ccccc}
\hline Situação & $\left(\mathbf{F H}_{\mathbf{0}}\right)$ & $\mathbf{t}_{\overline{\mathrm{e}}}$ & $\mathbf{r}_{\mathrm{yjy} 1}$ & Decisão \\
1 & $\mathrm{~ns}$ & $\mathrm{~ns}$ & $\mathrm{r} \geq(1-|\overline{\mathrm{e}}|)$ & $\mathrm{Y}_{\mathrm{J}}=\mathrm{Y}_{1}$ \\
2 & $\mathrm{~ns}$ & $\mathrm{~ns}$ & $\mathrm{r} \leq(1-|\overline{\mathrm{e}}|)$ & $\mathrm{Y}_{\mathrm{J}} \neq \mathrm{Y}_{1}$ \\
3 & $\mathrm{~ns}$ & $*$ & $\mathrm{r} \geq(1-|\overline{\mathrm{e}}|)$ & $\mathrm{Y}_{\mathrm{J}} \neq \mathrm{Y}_{1}$ \\
4 & $\mathrm{~ns}$ & $*$ & $\mathrm{r} \leq(1-|\overline{\mathrm{e}}|)$ & $\mathrm{Y}_{\mathrm{J}} \neq \mathrm{Y}_{1}$ \\
5 & $*$ & $\mathrm{~ns}$ & $\mathrm{r} \geq(1-|\overline{\mathrm{e}}|)$ & $\mathrm{Y}_{\mathrm{J}} \neq \mathrm{Y}_{1}$ \\
6 & $*$ & $\mathrm{~ns}$ & $\mathrm{r} \leq(1-|\overline{\mathrm{e}}|)$ & $\mathrm{Y}_{\mathrm{J}} \neq \mathrm{Y}_{1}$ \\
7 & $*$ & $*$ & $\mathrm{r} \geq(1-|\overline{\mathrm{e}}|)$ & $\mathrm{Y}_{\mathrm{J}} \neq \mathrm{Y}_{1}$ \\
8 & $*$ & $*$ & $\mathrm{r} \leq(1-|\overline{\mathrm{e}}|)$ & $\mathrm{Y}_{\mathrm{J}} \neq \mathrm{Y}_{1}$ \\
\hline
\end{tabular}

Onde: ns = não significativo ao nível de $5 \%$ de probabilidade de erro; * $=$ significativo ao nível de $5 \%$ de probabilidade de erro; $\mathrm{FH}_{0}=$ teste $\mathrm{F}$ de Graybill; $\mathrm{t}_{\overline{\mathrm{e}}}=$ teste $t$ para erro-médio; $\mathrm{r}_{\text {yjy } 1}$ correlação linear; $\mathrm{Y}_{\mathrm{j}}=$ valores observados; $\mathrm{Y}_{1=}$ valores estimados. Fonte: Leite \& Oliveira (2002). 


\section{Resultados}

O tempo de varredura laser em cada unidade amostral, sem a realização dos registros fotográficos, foi de aproximadamente 7,50 min, independe do tamanho da unidade amostral (UA). Como resultado da delimitação, foi obtida uma área circular referente ao tamanho da unidade amostral (Figura 3).

$\mathrm{Na}$ Figura 3A são mostrados os dados brutos de uma varredura simples, podendo-se observar uma maior densidade de pontos próxima ao centro da varredura. À medida que aumenta a distância das árvores ao equipamento, a quantidade de pontos observados é menor. Nas unidades amostrais de 200 e $300 \mathrm{~m}^{2}$ (Figuras $3 \mathrm{~B}$ e $3 \mathrm{C}$ ) os pontos laser que chegam até às árvores da borda não são influenciados pela resolução de varredura, não alterando a densidade de pontos. Entretanto, na UA de $400 \mathrm{~m}^{2}$ (Figura 3D), as árvores da borda sofrem mais alterações, quando comparadas com as dos tamanhos menores, apesar do raio de 11,28 m (relativamente próximo ao equipamento).

Na Figura 4 são apresentados os detalhes da seção transversal no centro e na borda da unidade amostral.

Quando analisado o formato da seção transversal mais próxima ao equipamento e/ou quando diminui o tamanho da unidade amostral, os pontos laser referentes às árvores apresentam-se, geralmente, na forma de um semicírculo. As árvores da borda da unidade amostral (Figura 4B) tendem a conter menos pontos que as árvores mais próximas ao equipamento.

De acordo com os resultados médios de número de árvores detectadas por tamanho de unidade amostral (Tabela 4), constatou-se que em todas as idades

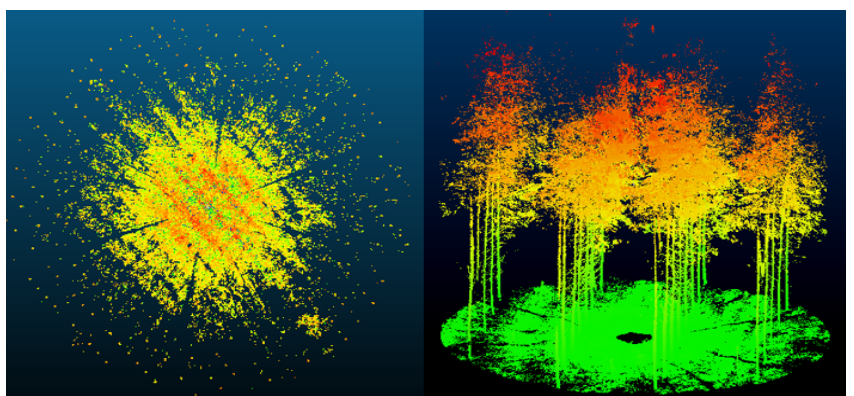

A)
B) analisadas foram encontrados erros na estimativa do número de árvores, exceto no talhão com 2 anos sem desrama, onde houve $100 \%$ de concordância nos tamanhos de unidades amostrais de 200 e $300 \mathrm{~m}^{2}$. Isto corrobora que a detecção automática proporciona melhores resultados em árvores com menores diâmetros e unidades amostrais com menores dimensões e, também, indica que a presença de galhos interfere na identificação das árvores.

Tabela 4. Porcentagem média de árvores detectadas automaticamente em unidades amostrais de 200, 300 e $400 \mathrm{~m}^{2}$ em plantios de Eucalyptus spp. em Três Lagoas, MS.

Table 4. Trees average percentage identified by automatic identification algorithm in sample units of 200,300 e $400 \mathrm{~m}^{2}$ in plantations of Eucalyptus spp in Três Lagoas, MS.

\begin{tabular}{cccc}
\hline Idade (anos) & $\mathbf{2 0 0} \mathbf{~ m}^{\mathbf{2}}$ & $\mathbf{3 0 0} \mathbf{~ m}^{\mathbf{2}}$ & $\mathbf{4 0 0} \mathbf{~ m}^{\mathbf{2}}$ \\
\hline 2 - sem desrama & 100,0 & 100,0 & 90,2 \\
2 - com desrama & 99,0 & 99,2 & 97,8 \\
4,5 & 96,9 & 95,2 & 90,6 \\
5,5 & 97,6 & 97,6 & 97,2 \\
\hline
\end{tabular}

A detecção das árvores nas unidades amostrais foi satisfatória (acima de 90\%) em todas as idades e tamanhos de unidades amostrais. Na Figura 5 são apresentados os resultados referentes à detecção das árvores nas unidades amostrais de diferentes dimensões.

O algoritmo detectou corretamente 100\% das árvores nas UA com 2 anos sem desrama, quando confrontadas com a contagem em campo, nas parcelas de 200 e $300 \mathrm{~m}^{2}$. Todavia, nas parcelas de $400 \mathrm{~m}^{2}$ o método automatizado subestimou o número de árvores.

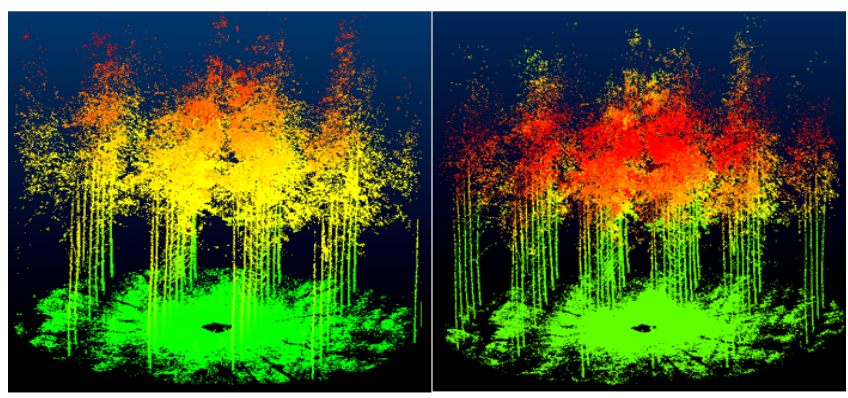

C)

Figura 3. Varredura simples em uma unidade amostral em povoamento de 2 anos. A) projeção da nuvem de pontos; B) unidade amostral de $200 \mathrm{~m}^{2}$; C) unidade amostral de $300 \mathrm{~m}^{2}$; D) unidade amostral de $400 \mathrm{~m}^{2}$.

Figure 3. Simple scan on a sample unit. A) projected point cloud; B) $200 \mathrm{~m}^{2}$ sample unit; C) $300 \mathrm{~m}^{2}$ sample unit; D) $400 \mathrm{~m}^{2}$ sample unit. 
Figura 4. A) resultado do processamento utilizando varredura simples em unidade amostral com $400 \mathrm{~m}^{2}$ em povoamento com 2 anos (sem desrama). B) Formato da seção transversal próxima ao equipamento. C) Formato da seção transversal na borda da unidade amostral.

Figure 4. A) processing result of $400 \mathrm{~m}^{2}$ sample unit using simple scan at stand of trees with 2 years-old. B) Cross-section shape close to the equipment. C) Cross-section shape at the sample unit edge.

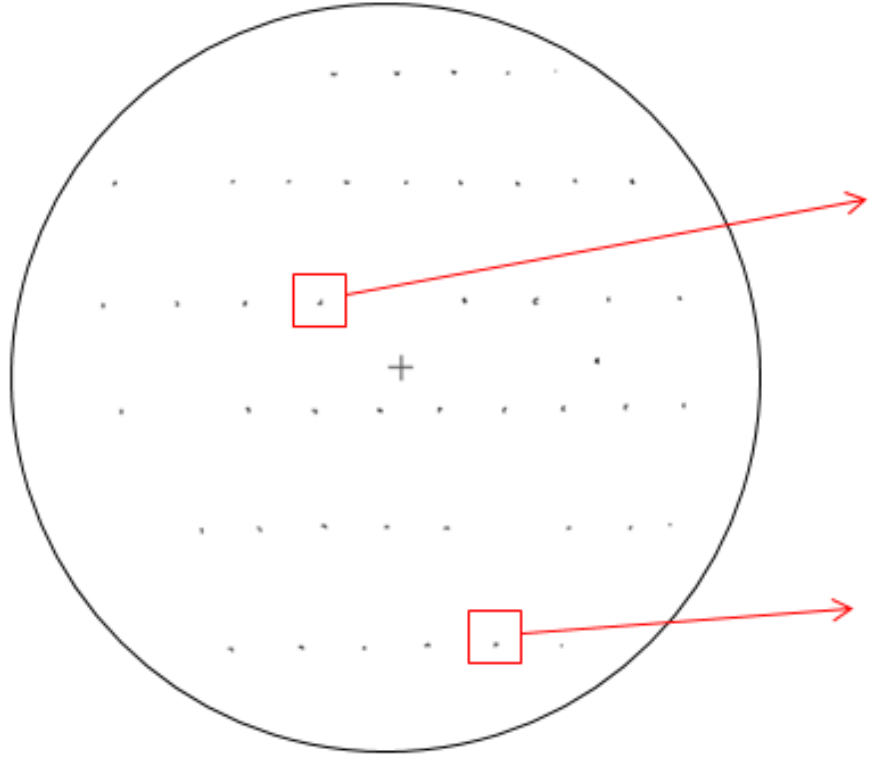

A

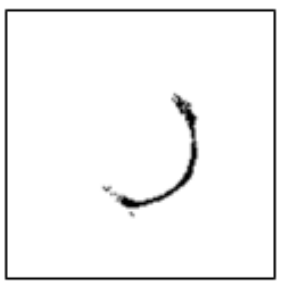

B

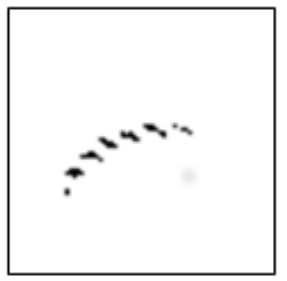

C
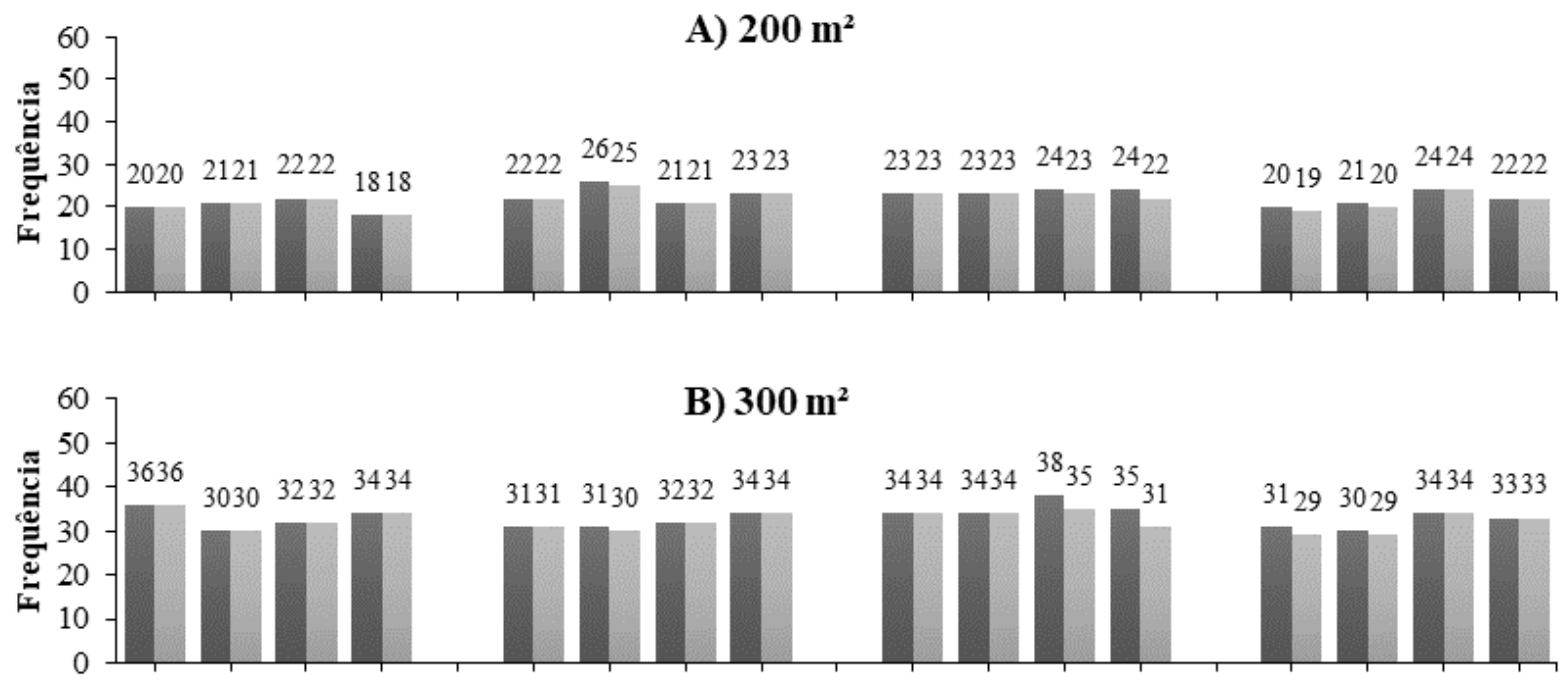

C) $400 \mathrm{~m}^{2}$

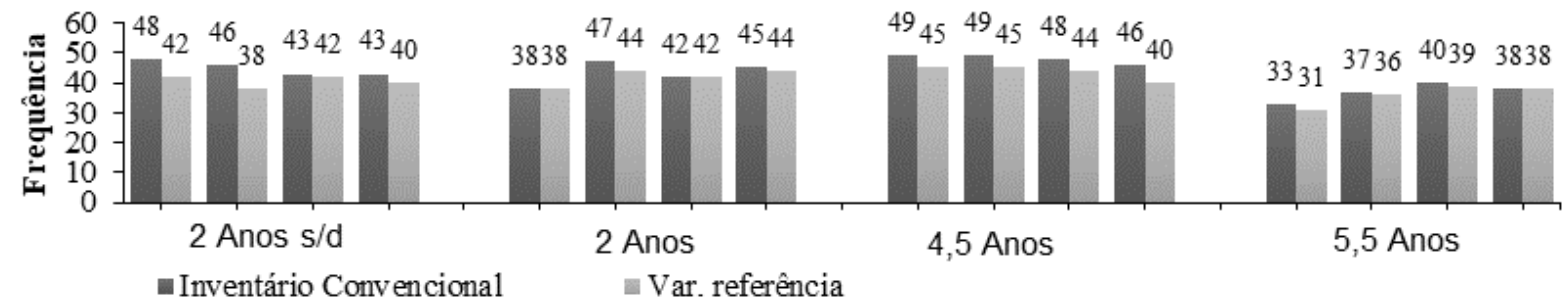

Figura 5. Árvores identificadas de maneira convencional e automaticamente, por idade de plantio de Eucalyptus spp. em Três Lagoas, MS, em diferentes tamanhos de unidades amostrais (200, 300 e $\left.400 \mathrm{~m}^{2}\right)$.

Figure 5. Trees detected by laser scanning and by conventional inventory by age in of Eucalyptus spp. plantation, in Três Lagoas, MS, for different sizes of sample units $\left(200,300\right.$ e $\left.400 \mathrm{~m}^{2}\right)$. 
Para a idade de 2 anos com desrama os resultados foram semelhantes, porém na área $200 \mathrm{~m}^{2}$ na unidade amostral 6, o método de detecção com laser identificou $95,4 \%$. Isto pode ser explicado por uma árvore ter ocultado outra devido ao alinhamento do plantio.

Os maiores erros de número de árvores identificadas foram nos talhões com árvores de 4,5 e 5,5 anos, respectivamente para unidades amostrais de $400 \mathrm{~m}^{2}$, onde não foi possível detectar todas as árvores. Árvores com 4,5 anos nas unidades amostrais com 200 e $300 \mathrm{~m}^{2}$ resultou em $100 \%$ de acerto. Nas unidades amostrais 9 e 10 de $300 \mathrm{~m}^{2}$ (Figura 5B) foi observada uma concordância de $100 \%$ e, consequentemente, também para a unidade amostral de $200 \mathrm{~m}^{2}$. No entanto, árvores maiores, com 5,5 anos, a detecção de 100\% das árvores foi possível em apenas uma unidade amostral (UA 16, de $400 \mathrm{~m}^{2}, 100 \%$ ).

Adotando o tamanho de $300 \mathrm{~m}^{2}$, houve acerto de $100 \%$ somente na unidade amostral 15. Para as demais unidades amostrais foram observados erros de detecção que permaneceram constantes nas unidades amostrais de $200 \mathrm{~m}^{2}$.
Para a coleta de dados utilizando o laser, a frequência de diâmetros maiores nas idades de 4,5 e 5,5 anos tende a ocultar mais árvores do que para a idade de $2 \operatorname{anos}(E$. grandis) que apresentam diâmetros menores.

$\mathrm{O}$ aigoritmo de detecção utiliza uma região de busca das árvores a uma distância mínima de $1,5 \mathrm{~m}$ préestabelecida de acordo com o espaçamento. Distâncias maiores subestimam o número de árvores, devido à inclusão dos pontos correspondentes aos galhos das árvores (Figura 6).

O resultado do teste L\&O, comparando os valores do número de árvores obtidos pelo inventário convencional e pelo algoritmo de detecção de árvores pode ser visto na Tabela 5. Pode-se verificar que, para todos os tamanhos de unidades amostrais, ocorreu rejeição de $\mathrm{H}_{0}$. Isso significa que houve diferenças significativas entre o número de árvores observado em campo e o detectado automaticamente pelo algoritmo.

De acordo com o teste estatístico L\&O com um nível de significância de $5 \%$, pode-se verificar que, para todos os tamanhos de unidades amostrais, ocorreu rejeição de $\mathrm{H}_{0}$. Isso significa que houve diferenças significativas entre o número de árvores observado em campo e o detectado automaticamente pelo algoritmo.

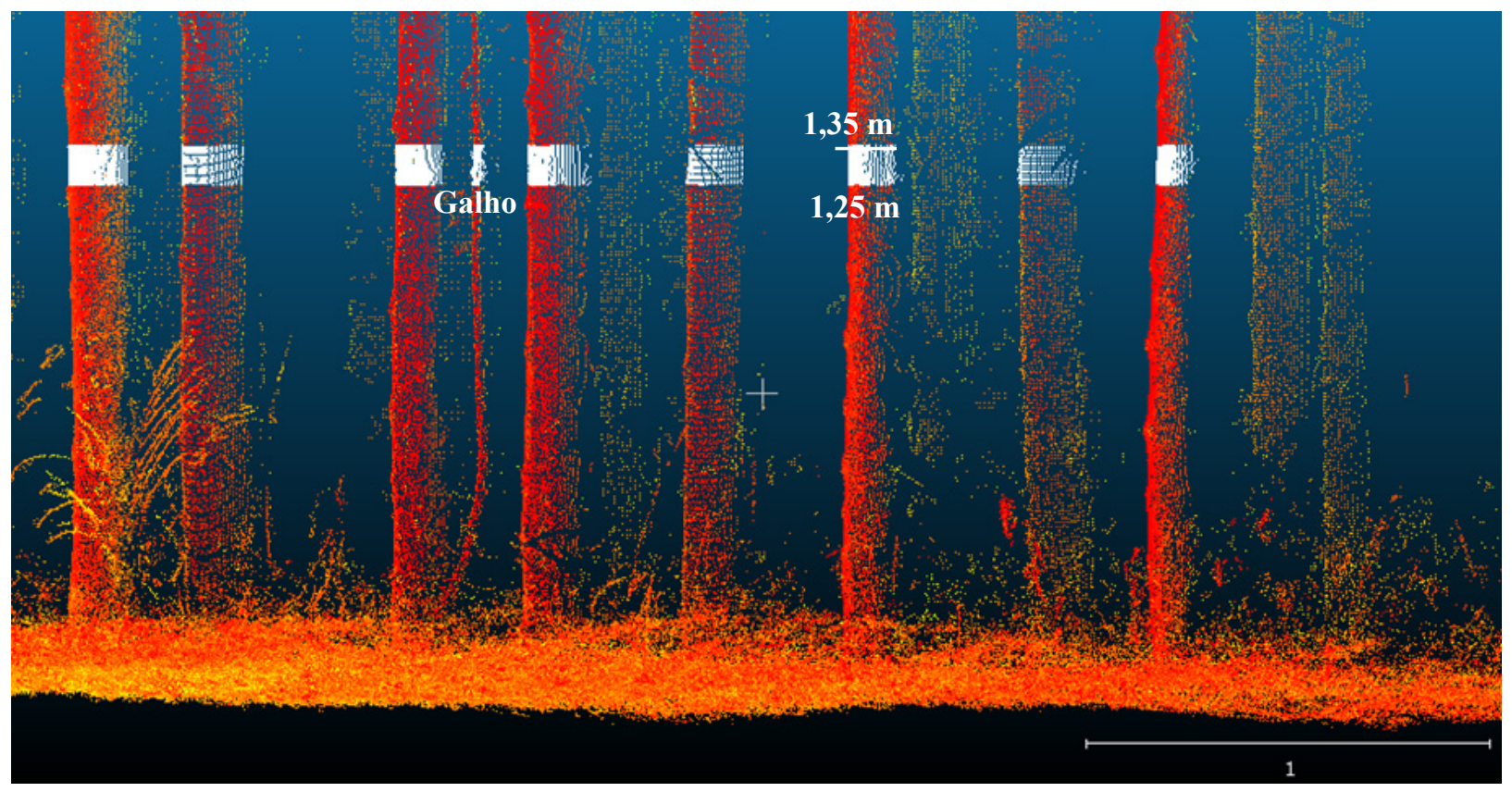

Figura 6. Comparação entre duas seções transversais de 0,10 m extraídas próximo a 1,30 m do solo (DAP), comparando uma árvore com galhos (esquerda) e sem galhos (direita) em plantios de Eucalyptus spp. com 4,5 anos.

Figure 6. Comparison between two 0.10-m-long cross sections taken near $1.30 \mathrm{~m}$ above ground level (DBH), comparing a tree with branches (left) and without branches (right) in plantations of Eucalyptus spp. with 4,5 years. 
Tabela 5. Análise estatística do número de árvores pelo teste estatístico L\&O, F de Graybill e t em unidades amostrais de 200, 300 e $400 \mathrm{~m}^{2}$ em plantios de Eucalyptus spp.

Table 5. Statistical analysis of the number of trees using L\&O test, Graybill's F and t-test in sampling units of 200, 300 and $400 \mathrm{~m}^{2}$ in plantations of.Eucalyptus spp.

\begin{tabular}{cccccccc}
\hline U. A. $\left(\mathbf{m}^{2}\right)$ & $\mathbf{F}\left(\mathbf{H}_{\mathbf{o}}\right)$ & $\mathbf{t}_{\overline{\mathrm{e}}}$ & $\mathbf{r}_{\text {yjy } 1} \geq|\mathbf{1}-\overline{\mathbf{e}}|$ & $\mathbf{R}^{2}$ & $\mathbf{C V} \%$ & Decisão \\
\hline 200 & $4.038^{\text {ns }}$ & $2.448^{\text {ns }}$ & Não & 0.9005 & 2.7678 & $\mathrm{Yj} \neq \mathrm{Y} 1$. \\
300 & $3.289^{\text {ns }}$ & $2.267^{\text {ns }}$ & Não & 0.7063 & 3.78953 & $\mathrm{Yj} \neq \mathrm{Y} 1$. \\
400 & $21.058^{*}$ & $4.706^{*}$ & Não & 0.7574 & 4.77652 & $\mathrm{Yj} \neq \mathrm{Y} 1$. \\
\hline
\end{tabular}

Onde: ns = não significativo ao nível de $5 \%$ de probabilidade de erro; $*$ = significativo ao nível de $5 \%$ de probabilidade de erro; U.A. $=$ Unidade amostral; F $(\mathrm{H} 0)=$ teste $\mathrm{F}$ de Graybill; $\mathrm{t}_{\mathrm{e}}=$ teste $t$ para erro-médio; $\mathrm{r}_{\mathrm{yjy} 1}$ correlação linear; $\mathrm{R}^{2}=$ coeficiente de correlação; $\mathrm{CV}=$ coeficiente de variação $(\%) ; \mathrm{Y}_{\mathrm{j}}=$ valores observados; $\mathrm{Y}_{1=}$ valores estimados.

$\mathrm{O}$ resultado do teste $\mathrm{F}$ de Graybill, para as unidades amostrais de 200 e $300 \mathrm{~m}^{2}$ foi não significativo, ou seja, os valores observados são iguais estatisticamente aos valores encontrados pelo algoritmo. A não significância para os tamanhos de 200 e $300 \mathrm{~m}^{2}$ também foi constatada pelo teste t. Para as unidades amostrais de $400 \mathrm{~m}^{2}$, os valores de $\mathrm{F}\left(\mathrm{H}_{0}\right)$ e t foram significativos, confirmando a diferença entre a verdade de campo e os resultados da detecção automática.

Pode-se verificar, também, que as unidades amostrais de $200 \mathrm{~m}^{2}$ apresentaram maior correlação $(\mathrm{R}=0,9)$ entre o número de árvores observado e o detectado pelo algoritmo em comparação com os demais tamanhos. O CV\% também foi menor para as unidades amostrais de $200 \mathrm{~m}^{2}(2,76 \%)$, em relação às unidades amostrais de 300 e $400 \mathrm{~m}^{2}$ (3,78\% e 4,77\%, respectivamente). Sendo assim, as unidades amostrais de $200 \mathrm{~m}^{2}$ apresentam os menores erros utilizando varreduras simples.

Algumas árvores ficaram ocultas, devido ao sombreamento, ocasionado principalmente pelas árvores mais próximas ao equipamento (Figura 7).

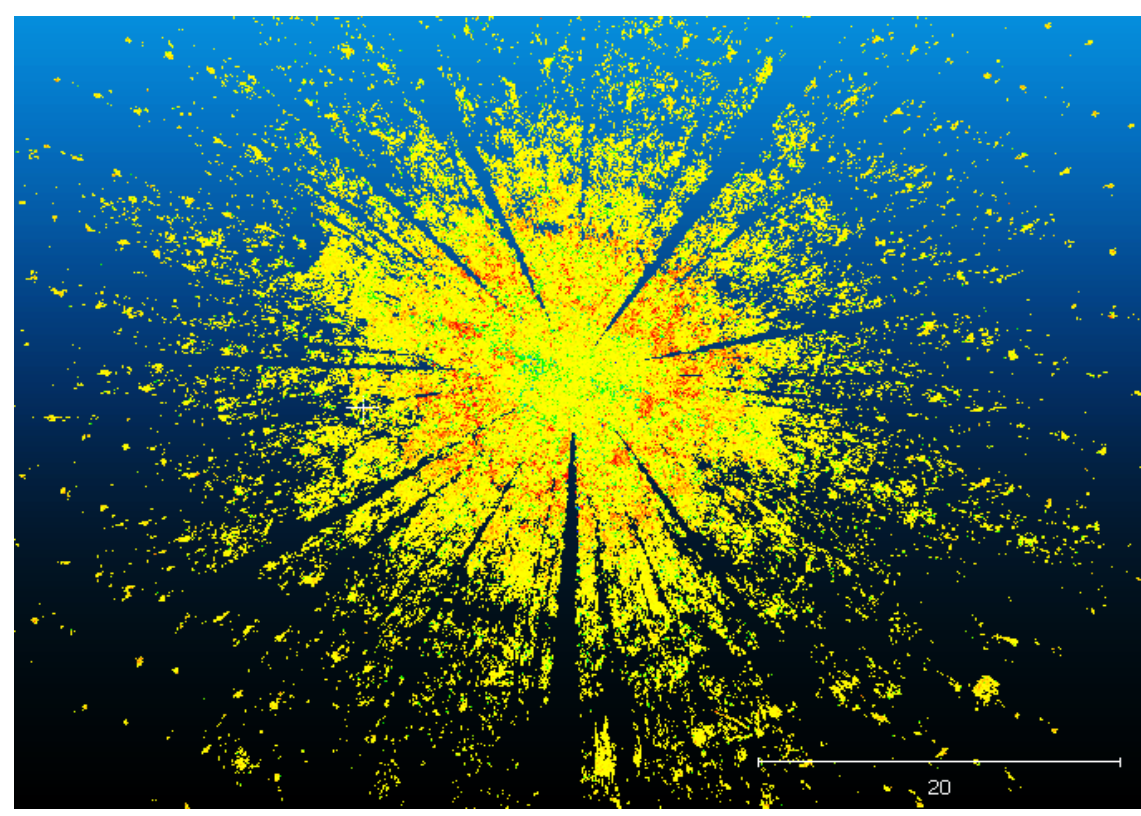

Figura 7. Unidade amostral em povoamento com 4,5 anos, mostrando o efeito de sombreamento ocasionado por árvores próximas ao equipamento.

Figure 7. Sampling unit in a 4.5 year-old stand, showing the shadow effefc caused by trees near the equipment. 


\section{Discussão}

O sombreamento formado pelas árvores próximas do equipamento laser aumentou na direção da borda da unidade amostral (Figura 7). O efeito do sombreamento é um fator que afeta a acurácia das estimativas do número de árvores em parcelas, principalmente nos maiores tamanhos, aumentando o risco de erros sistemáticos associados à subestimação do número de árvores existentes (Zasada et al., 2013; Buck et al., 2017).

Em parcelas menores, o efeito do sombreamento não sofre grande influência, pois menos árvores são consideradas na amostra. Dessa forma, quanto maior a unidade amostral e maior o diâmetro das árvores, maior será o efeito de sombreamento. Caso o alinhamento seja regular entre árvores e entre as linhas (sem curvas de nível), há um aumento do número de árvores que ficarão ocultas e, consequentemente, suas informações não podem ser obtidas pela varredura laser. O efeito do sombreamento depende da estrutura vertical e horizontal dos povoamentos e características do local, como a topografia (Pueschel, 2013; Buck et al., 2017).

Buck et al. (2017) indicaram subestimativas quanto ao número de árvores interpretadas visualmente em nuvens de pontos de parcelas circulares com área de $400 \mathrm{~m}^{2}$ levantadas por varredura simples, em que foram identificadas em média 95\% das árvores para idade de 5 anos e $96 \%$ para as idades de 4 e 2 anos, resultados estes similares aos obtidos na presente pesquisa. A vantagem em se utilizar varredura simples está na rapidez na coleta dos dados em relação ao inventário convencional. Ainda, a varredura laser capta não somente o diâmetro e altura das árvores da parcela, mas dissemina pontos por todo o tronco da árvore, podendo ser realizada a modelagem sem a derrubada.

As características do equipamento laser (por diferença de fase ou time-of-flight) e a resolução do equipamento influenciam na detecção das árvores. A densidade de pontos $3 \mathrm{D}$ diminui à medida que aumenta o tamanho da unidade amostral (UA), tornando-se inviável a adoção de parcelas maiores que $400 \mathrm{~m}^{2}$ quando se utiliza varredura simples (Li \& Weiskittel, 2014). Estes mesmos autores avaliaram um algoritmo de detecção automática em um experimento com árvores espaçadas regularmente $(1,29$ x 1,29 m) em que $98 \%$ das árvores na unidade amostral de $666,7 \mathrm{~m}^{2}$ foram corretamente detectadas, e os $2 \%$ não detectados foram árvores ocluídas por outras árvores.
A alta precisão de detecção foi relatada em estudos iniciais apenas em florestas com baixa densidade de árvores. Liang (2013) comentou que a taxa de detecção é acima de $80 \%$ em UA com densidade menor do que 410 árvores ha ${ }^{-1}$. A precisão de detecção é relatada como $59-72 \%$ em florestas com densidade de 735 árvores ha-1

Os resultados listados na Tabela 6 resumem o estado da arte da pesquisa sobre este tema. No entanto, eles não devem ser entendidos como uma comparação rigorosa, porque os resultados dependem das espécies e porte das árvores, da densidade, do tipo de floresta, do instrumento utilizado e dos métodos de processamento. Outro fator relacionado a detecção de árvores é a topografia, porém, quando adotadas unidades amostrais menores (Liang, 2013) esse efeito é praticamente nulo, caso contrário, a topografia acentuada causa menor taxa de detecção.

Os resultados da presente pesquisa demostraram boa acurácia na detecção em comparação com as demais pesquisas mencionadas anteriormente, devido principalmente à distribuição espacial de árvores na parcela. Em florestas boreais ou de coníferas da Europa, não há espaçamento regular entre as árvores e as unidades amostrais geralmente são maiores, acarretando maior efeito de sombreamento quando se utiliza varredura simples.

O instrumento usado também pode influenciar o resultado. Strahler et al. (2008) e Lovell et al. (2011) obtiveram as taxas de detecção (Tabela 6) um pouco menores nas unidades amostrais de baixa densidade. $\mathrm{O}$ instrumento utilizado por esses autores é baseado na diferença de fase, similar ao utilizado no presente trabalho. Segundo Liang (2013), os resultados são mais relacionados ao mecanismo de medição do scanner.

Conforme Liang et al. (2012), a aplicação do algoritmo para a identificação do fuste não é dependente do conhecimento da estrutura e formato dos dados. $\mathrm{Na}$ pesquisa descrita por esses autores, usando nove unidades amostrais (raio $=10 \mathrm{~m}$ ), muito próximas dos tamanhos das unidades amostrais adotadas neste estudo, obteve-se $73 \%$ de acurácia de identificação das árvores.

Na gestão de povoamentos florestais densos, a maioria das árvores pode ser localizada usando varreduras simples, mas o emprego de varreduras múltiplas e de uma edição de dados interativa da nuvem de pontos precisa ser mais estudado para proporcionar um sistema prático e viável para diferentes ambientes florestais (Liang et al., 2012). 
Tabela 6. Precisão de identificação de árvores para varredura simples relatada em estudos anteriores em comparação com os resultados obtidos nesta pesquisa em plantios de Eucalyptus spp. em Três Lagoas, MS.

Table 6. Tree identification accuracy for single scan reported in previous studies compared with the results obtained in this research in plantations of Eucalyptus spp. in Três Lagoas, MS.

\begin{tabular}{|c|c|c|c|c|c|c|}
\hline & \multirow[b]{2}{*}{$\mathbf{N}^{\circ}$ de UA } & \multirow[b]{2}{*}{ Tipo de floresta } & \multicolumn{3}{|c|}{ Unidades amostrais } & \multirow{2}{*}{$\begin{array}{c}\text { Detecção } \\
\%\end{array}$} \\
\hline & & & Raio (m) & Área $\left(m^{2}\right)$ & Árvores ha' ${ }^{-1}$ & \\
\hline Thies \& Spiecker (2004) & 1 & Floresta Boreal & - & 900 & 555,6 & 22 \\
\hline Maas et al. (2008) & 3 & Floresta Boreal & 15 & 707 & $212-410$ & $86.7-100$ \\
\hline Strahler et al. (2008) & 1 & Pinus ponderosa & 50 & 7854 & 130 & 40,2 \\
\hline Brolly \& Kiraly (2009) & 1 & $\begin{array}{l}\text { Quercus petraea } \\
\text { Fagus sylvatica } \\
\text { Picea abies }\end{array}$ & 30 & 2827 & 753 & $62,9-72,3$ \\
\hline \multirow{2}{*}{ Murphy et al. (2010) } & 18 & \multirow{2}{*}{ Pinus radiata } & - & 1000 & $207-570$ & 59 \\
\hline & 15 & & $10-20$ & $314-1257$ & $153-326$ & 82 \\
\hline Lovell et al. (2011) & 2 & Pinus ponderosa & $20-50$ & $1257-7854$ & $124 / 477$ & $54 / 68$ \\
\hline Liang et al. (2012) & 9 & $\begin{array}{c}\text { Pinus sylvestris } \\
\text { Picea abies }\end{array}$ & 10 & 314 & $509-1432$ & 73 \\
\hline Liang (2013) & 5 & $\begin{array}{l}\text { Pinus sylvestris } \\
\text { Picea abies }\end{array}$ & 10 & 314 & $605-1210$ & 73,4 \\
\hline \multirow{3}{*}{ Presente pesquisa } & \multirow{3}{*}{16} & & 7,98 & 200 & $827-1262$ & 98,3 \\
\hline & & Eucalyptus spp. & 9,77 & 300 & $827-1262$ & 98 \\
\hline & & & 12,28 & 400 & $827-1262$ & 93,9 \\
\hline
\end{tabular}

\section{Conclusão}

O algoritmo mostrou-se eficiente na detecção automática em plantios de Eucalyptus spp., porém é necessário o ajuste da região de busca de acordo com o espaçamento, para não ocorrer subestimativa no número de árvores na parcela.

O uso de varredura simples com laser terrestre em parcelas circulares de $200 \mathrm{~m}^{2}$ e $300 \mathrm{~m}^{2}$ para a idade de 2 anos sem desrama, obtiveram uma taxa de detecção de $100 \%$. Para os plantios com 4,5 e 5,5 anos o efeito de sombreamento é maior em relação aos plantios mais jovens, pois os maiores diâmetros causam oclusão, principalmente das árvores de borda.

Para diminuir ou excluir o efeito de sombreamento, sugere-se que sejam testadas varreduras múltiplas, porém, acarretaria maior tempo de levantamento em campo, sendo imprescindível um estudo de viabilidade.

\section{Agradecimentos}

Os autores agradecem a Eldorado do Brasil pelo apoio ao projeto de pesquisa e a Santiago \& Cintra pela disponibilização do equipamento Trimble TX5 e suporte técnico à coleta de dados.

\section{Referências}

Bienert, A. et al. Application of terrestrial laser scanners for the determination of forest inventory parameters. International Archives of Photogrammetry, Remote Sensing and Spatial Information Sciences, v. 36, 2006.

Buck, A. L. B. Análise de métodos de filtragem em dados laser scanner terrestre aplicados à modelagem tridimensional do tronco de Pinus spp. 2012. 135 f. Dissertação (Mestrado em Engenharia Florestal) - Universidade Federal do Paraná, Curitiba.

Buck, A. L. B. et al. Avaliação da modelagem tridimensional do tronco de Pinus elliottii a partir de dados LASER terrestre. Ambiência, v. 8, p. 549-557, 2012.

Buck, A. L. B. et al. Detecção de árvores em nuvens de pontos de varredura laser terrestre. Boletim de Ciências Geodésicas, v. 23, n. 1, p. 21-38, 2017.

Brolly, G. \& Kiraly, G. Algorithms for stem mapping by means of terrestrial laser scanning. Acta Silvatica et Lignaria Hungarica, n. 5, p. 119-130, 2009.

Graybill, F. A. Theory and application of the linear model. Belmont: Duxbury Press, 1976. 704 p. 
Henning, G. H. \& Radtke, P. J. Ground-based laser imaging for assessing three dimensional forest canopy structure. Photogrammetric Engineering \& Remote Sensing, v. 72, n. 12, p. 1349-1358, 2006. http://dx.doi.org/10.14358/PERS.72.12.1349.

Király, G. \& Brolly, G. Volume calculations of single trees based on terrestrial laser scanning. In: SILVILASER: INTERNATIONAL CONFERENCE ON LIDAR APPLICATIONS FOR ASSESSING FOREST ECOSYSTEMS, 10,, 2010, Freiburg. Proceedings... Freiburg: Forstliche Versuchs-und Forschungsanstalt, BadenWurtenberg, 2010. CD-ROM.

Leite, H. G. \& Oliveira, F. H. T. Statistical procedure to test the identity of analytical methods. Communications in Soil Science and Plant Analysis, v. 33, n. 7, 2002. http://dx.doi.org/10.1081/ CSS-120003875.

Liang, X. et al. Automatic stem mapping using single-scan terrestrial laser scanning. IEEE Transactions on Geoscience and Remote Sensing, v. 50, n. 2. p. 661-670, 2012. http://dx.doi.org/10.1109/ TGRS.2011.2161613.

Liang, X. \& Hyyppä, J. Detecting changes in forest structure over time with bi-temporal terrestrial laser scanning data. ISPRS International Journal of Geo-Information, v. 1, n. 3, p. 242-255, 2013. http://dx.doi.org/10.3390/ijgi1030242.

Liang, X. Feasibility of Terrestrial Laser Scanning for Plot wise Forest Inventories. 2013. 150 f. Tese (Doctoral of Science in Technology) - Kirkkonummi, Finland.

Lingnau, C. et al. Determinação de diâmetros a diferentes alturas de árvores individuais a partir de dados laser terrestre. In: SIMPÓSIO BRASILEIRO DE SENSORIAMENTO REMOTO, 14., 2009, Natal. Anais... São José dos Campos: INPE, 2009.

Lingnau, C. et al. Modelagem do fuste em povoamentos de Pinus taeda a partir da varredura laser terrestre. In: SEMINÁRIO DE ATUALIZAÇÃO EM SENSORIAMENTO REMOTO E SISTEMAS DE INFORMAÇÕES GEOGRÁFICAS APLICADOS À ENGENHARIA FLORESTAL, 9., 2010, Curitiba. Anais... Curitiba: FUPEF, 2010. p. 111-118.

Li, R. \& Weiskittel, A. R. Comparison of model forms for estimating stem taper and volume in the primary conifer species of the North American Acadian Region. Annals of Forest Science, v. 67, n. 3, p. 302, 2014. http://dx.doi.org/10.1051/forest/2009109.

Lovell, J. L. et al. Measuring tree stem diameters using intensity profiles from ground-based scanning lidar from a fixed viewpoint. ISPRS Journal of Photogrammetry and Remote Sensing, v. 66, n. 1, p. 46-55, 2011.
Maas, H. G. et al. Automatic forest inventory parameter determination from terrestrial laser scanner data. International Journal of Remote Sensing, v. 29, n. 5, p. 1579-1593, 2008. http://dx.doi.org/10.1016/j. isprsjprs.2010.08.006.

Maia, T. C. B. Estudo e análise de poligonais segundo a NBR 13.133 e o sistema de posicionamento global. 1999. Dissertação (Mestrado em Transportes) - Universidade de São Paulo, São Carlos.

Martins Neto, R. P. et al. Avaliação da varredura laser terrestre em diferentes distâncias da árvore para mensurar variáveis dendrométricas. Boletim de Ciências Geodésicas, v. 19, n. 3, p. 420433, 2013. http://dx.doi.org/10.1590/S1982-21702013000300005.

Murphy, G. E. et al. Tree value and log product yield determination in radiata pine (Pinus radiata) plantations in Australia: comparisons of terrestrial laser scanning with a forest inventory system and manual measurements. Canadian Journal of Forest Research, v. 40, p. 2223-2233, 2010. http://dx.doi.org/10.1139/X10-171.

Pueschel, P. et al. The influence of scan mode and circle fitting on tree stem detection, stem diameter and volume extraction from terrestrial laser scans. ISPRS Journal of Photogrammetry and Remote Sensing, v. 77, p. 44-56, 2013. http://dx.doi.org/10.1016/j. isprsjprs.2012.12.001.

Silva, M. N. Aplicação de laser scanner terrestre para determinação de variáveis dendrométricas em Pinus spp. 2011. 126 f. Dissertação (Mestrado em Engenharia Florestal) Universidade Federal do Paraná, Curitiba.

Simonse, M. et al. Automatic determination of forest inventory parameters using terrestrial Laser scanning. Proceedings of the Scand Laser Scientific Workshop on Airborne Laser Scanning of Forests, p. 251-257, 2003.

Strahler, A. H. et al. Retrieval of forest structural parameters using a ground-based lidar instrument (Echidna $\AA$ ). Canadian Journal of Remote Sensing, v. 34, p. S426-S440, 2008. http://dx.doi. org/10.5589/m08-046.

Thies, M. \& Spiecker, H. Evaluation and future prospects of terrestrial laser scanning for standardized forest inventories. International Archives of Photogrammetry, Remote Sensing and Spatial Information Sciences, v. 36, p. 192-197, 2004.

Trochta, J. et al. Arrangement of terrestrial laser scanner positions for area-wide stem mapping of natural forests. Canadian Journal of Forest Research, v. 43, n. 999, p. 355-363, 2013. http://dx.doi. org/10.1139/cjfr-2012-0347.

Zasada, M. et al. Horizon visibility and accuracy of stocking determination on circular sample plots using automated remote measurement techniques. Forest Ecology and Management, v. 302, p. 171-177, 2013. http://dx.doi.org/10.1016/j.foreco.2013.03.041. 